\title{
Norois
}

Environnement, aménagement, société

\section{Les impacts d'un parc naturel régional (PNR) sur les évolutions socio-démographiques de son espace rural : le cas du Vexin français}

Socio-demographics consequences of the Creation of a Natural Regional Park: the Example of le Vexin français

\section{Didier Desponds}

\section{OpenEdition Journals}

Édition électronique

URL : https://journals.openedition.org/norois/1620

DOI : $10.4000 /$ norois. 1620

ISBN : 978-2-7535-1550-5

ISSN : $1760-8546$

Éditeur

Presses universitaires de Rennes

\section{Édition imprimée}

Date de publication : 1 mars 2007

Pagination : 47-60

ISBN : 978-2-7535-0457-8

ISSN : 0029-182X

Référence électronique

Didier Desponds, «Les impacts d'un parc naturel régional (PNR) sur les évolutions socio-

démographiques de son espace rural : le cas du Vexin français », Norois [En ligne], 202 | 2007/1, mis en ligne le 01 mars 2009, consulté le 13 janvier 2022. URL : http://journals.openedition.org/norois/ 1620 ; DOI : https://doi.org/10.4000/norois. 1620 


\title{
LES IMPACTS D'UN PARC NATUREL RÉGIONAL (PNR)
}

\section{SUR LES ÉVOLUTIONS SOCIO-DÉMOGRAPHIQUES DE SON ESPACE RURAL : LE CAS DU VEXIN FRANÇAIS}

\author{
DIDIER DESPONDS \\ MrTe - EA 1896 CNRS \\ (Université de Cergy-Pontoise), \\ 33, boulevard du Port -95011 Cergy-Pontoise cedex \\ didier.desponds@lsh.u-cergy.fr
}

\section{RÉSUMÉ}

La préservation des espaces ruraux franciliens constitue un défi majeur. La création de quatre PNR (Parcs Naturels Régionaux) se fuxait plusieurs objectifs : contrôler la croissance démographique, limiter les nouvelles constructions, préserver les paysages et promouvoir l'activité agricole. À travers l'exemple du PNR du Vexin français, vaste territoire implanté au nord de l'agglomération parisienne, au contact de la Ville Nouvelle de Cergy-Pontoise, apparaît une réalité plus complexe : amorce d'un processus d'embourgeoisement et dépendance économique croissante à l'encontre des pôles périphériques.

Mots CLÉS : Filtrage social - Gentrification - Identité territoriale - Mobilités alternantes - Périurbanisation.

\footnotetext{
ABSTRACT

Socio-demographics consequences of the Creation of a Natural Regional Park. The Example of le Vexin français

Preserving rural spaces within Paris metropolitan area constitutes a major planning stake. In order to control urban sprawl, four Regional Natural Parks have been created since the 80s. The goals of the containment policies were: controlling demographic increase, restricting the building rules, preserving the landscapes and supporting the agricultural activities. The example of the Regional Natural Park of le Vexin Français, in the Northern vicinity of Paris, near the new town of Cergy-Pontoise, underlines what has been happening: gentrification's process has been developing in the area and meantime the economic activity has been decreasing.
}

KEY WORDS : Commuting Mobilities - Filtering Process - Gentrification - Peri-urban Sprawl - Territorial Identity.

Depuis la création précoce en décembre 1985 du PNR de la Haute vallée de Chevreuse, une ceinture de parcs se constitue progressivement autour de l'agglomération parisienne : PNR du Vexin français (créé en mai 1995), PNR du Gâtinais (mai 1999) et plus récemment, PNR Oise 
- Pays-de-France (mai 2004) ${ }^{1}$. Ces projets rendus possibles depuis le décret $n^{\circ} 67-158$ du $1^{\mathrm{er}}$ mars 1967 signé par le Général de Gaulle, sont soutenus par des maires locaux avec l’appui des Conseils régionaux. Ces derniers assurent en moyenne $40 \%$ des financements des PNR quand les soutiens des autres collectivités (départements, communes) ne s'élèvent qu’à $30 \%$.

Si quarante deux PNR sont recensés à ce jour par la Fédération française des Parcs naturels régionaux ${ }^{2}$, tous ne répondent pas aux mêmes logiques. Pour la grande majorité d'entre eux, il s'agit certes de préserver des espaces essentiellement "naturels », mais aussi de compenser les effets de la déprise agricole et du dépeuplement. Dans le jeu de la concurrence entre les territoires, le label PNR vise à les « extirper d'une spirale dépréciative », en engendrant une dynamique de valorisation le plus souvent axée sur le tourisme vert. Le cas des PNR périurbains est a priori bien différent. C'est davantage la perception d'une pression urbaine à repousser, ou à canaliser qui va prévaloir. Le PNR du Vexin français est à cet égard emblématique. Comme le signale le géographe J.-C. Cavard (Cavard, 1994), c'est le développement de la Ville Nouvelle de Cergy-Pontoise et ses perspectives d'expansion qui suscita une puissante mobilisation des élus locaux et d'associations fortement outillées, et en tout premier lieu celle des Amis du Vexin français. Acteur majeur de cette dernière, le démographe $\mathrm{J}$. Dupâquier ${ }^{3}$ insiste sur la nécessité qu'il y avait à maîtriser l'urbanisation afin de patrimonialiser les paysages et sur l'intense travail de sensibilisation qu'il fallut mettre en œuvre auprès des multiples décideurs territoriaux. Le président du Conseil général, A. Chauvin, joua dans ce domaine un rôle de catalyseur.

La charte du PNR ${ }^{4}$ est actuellement en cours de révision, le processus devant s'achever en 2007. Le comité syndical du Parc a validé le 18 septembre 2006, le projet de charte tel qu'il sera soumis à l'enquête d'utilité publique. Dans ce contexte, il est tentant d'évaluer l'impact des réglementations mises en ouvre sur ce vaste territoire, englobant 94 communes, pour une superficie de 66000 hectares et une population évaluée à environ 79000 personnes en 1999. Si le bilan concernant les aménagements ou les protections environnementales est connu et fortement médiatisé, il n'en va pas de même des mutations sociales que le PNR a enregistrées, ni des processus de valorisation immobilière et foncière. Celles-ci résultent-elles des règles strictes imposées par la charte du parc et les outils d'aménagement qui en découlent? Si un PNR situé dans une zone qualifiée de « rural profond » peut être perçu comme la moins mauvaise des façons de préserver de la vie dans un territoire en cours de désertification, un PNR en marge d'agglomération peut être appréhendé comme un mode de «sélection douce " des populations? Dans un contexte de demande résidentielle élevée pour ce type de localisation et d'offre particulièrement restreinte, les prix de l'immobilier s'y apprécient en effet, déclenchant des phénomènes de «filtrage social » des ménages (Desponds, 2003, 2005). Les contrepoids mis en œuvre, en termes d'habitat social, sont quant à eux extrêmement fragiles. Si les données statistiques ${ }^{5}$ mises à disposition par l'INSEE nous serviront de matière première, il en sera de même des données fiscales ${ }^{6}$ et de la base $\mathrm{BIEN}^{7}$. Cette dernière permet d'appréhender les logiques d'acquisitions immobilières effectuées par les ménages.

\footnotetext{
1. Le Syndicat mixte d'études et de programmation pour l'élaboration du projet de Charte du PNR des boucles de la Marne et de l'Ourcq ayant été dissous en février 2003, celui-ci ne devrait pas voir le jour, tout du moins à brève échéance. A propos des PNR périurbains, voir les analyses du Sénateur G. Larcher (1998).

2. Voir son site: [http://www.parcs-naturels-regionaux.tm.fr/].

3. Voir le portrait de J. Dupâquier réalisé dans le nº 4 de «Couleurs du Vexin ». avril 1998.

4. Charte approuvée le 9 mai 1995.

5. Données obtenues auprès de l'INSEE et du Centre Quetelet [http://www.centre.quetelet.cnrs.fr], habilité à diffuser depuis le 8 décembre 2001, les données de l'INSEE auprès des organismes de recherche.

6. Celles-ci furent rendues accessibles par les Hôtels des impôts du Val-d'Oise et des Yvelines.

7. Base d'Informations Économiques Notariales, base gérée par le Bureau Van-Dijk jusqu'en début 2006, depuis la diffusion en est directement assurée par Paris Notaires Services (voir [http://www.paris.notaires.fr]. Dans le cadre de cette étude, la base fut « gracieusement mise à disposition » par l'ADIL 95.
} 


\section{Face à la «Grande peur » urbaine, l'élaboration d'un PNR}

Les conditions dans lesquelles le Parc s'est initialement constitué présentent à cet égard un indéniable intérêt. Les spécificités locales incitent à porter attention à la relation qui s'est instaurée entre les deux entités les plus puissamment structurantes et a priori partiellement antagonistes, la ville nouvelle de Cergy-Pontoise d'une part, et le PNR du Vexin français de l'autre. La première a souvent été perçue comme une greffe dont il fallait à tout prix limiter la capacité de nuisance, les velléités, supposées ou réelles, d'expansion. La charte du parc, dans son préambule, se réfère explicitement à cette donne territoriale : «En région Île-de-France, l'équilibre est fragilisé par la poussée de l'urbanisation sur les espaces ruraux. La ville nouvelle de Cergy-Pontoise ainsi que l'urbanisation de la basse vallée de la Seine ont empiété sur les espaces naturels et agricoles du Vexin français. La demande croissante en logements individuels et en espaces verts de proximité renforce la pression urbaine sur les espaces ruraux. » Ces éléments de constat vont conduire à la mise en place d'un outillage réglementaire inscrit dans la charte du PNR et s'imposant, par l'entremise des POS (ultérieurement PLU, voir loi 2000-1208 du 13-12-2000) aux communes ayant opté pour leur intégration. Ainsi, l'article 7 indique : «La loi (cf. loi n 93-24 du 8 janvier 1993) sur la protection et la mise en valeur des paysages précise que les "documents d'urbanisme doivent être compatibles avec les orientations et mesures de la charte”. Les communes s'engagent à un développement démographique maîtrisé. Celui-ci en moyenne ne devra pas dépasser 0,75\% par an sur l'ensemble du territoire du Parc $^{8}$. » Un tel objectif est justifié plus loin par la nécessité de préserver des paysages (article 11) : «L'essentiel est de veiller à préserver les spécificités du Vexin et d'en développer les aspects positifs. Il faut éviter sa "rurbanisation", c'est-à-dire la banalisation et la perte d'identité des paysages ruraux consécutive à l'implantation des grandes infrastructures, aux urbanisations tentaculaires induites par ces infrastructures, aux extensions urbaines mal maîtrisées, au mitage des espaces naturels, au fractionnement et à l'aliénation des grands domaines. » Le terme de «rurbanisation » ici utilisé, ne l'est pas dans son acception sociale ${ }^{9}$, mais uniquement dans sa dimension paysagère qui peut d'ailleurs sembler contradictoire, " rurbanisation » rimant davantage avec la préservation d'un cadre environnemental rural. Le moyen permettant d'atteindre l'objectif fixé, est détaillé dans l'article 7.3. de la charte : «Les communes s'engagent à construire en priorité dans les centres urbains, à implanter les constructions neuves sous forme de greffe en continuité du bâti existant dans le cadre d'un développement modéré des bourgs, villages et hameaux et à maintenir des zones non constructibles en bordure des massifs boisés et des rivières, selon les prescriptions architecturales de la charte paysagère communale et selon le plan du Parc. » Concrètement ceci conduira à privilégier la densification des espaces bâtis plutôt que leur extension. La réhabilitation de corps de ferme, dans un contexte de disparition d'exploitations agricoles ${ }^{10}$ a permis d'offrir une réponse partielle à la demande de logements.

La nécessaire complémentarité entre les territoires était considérée comme indispensable par J.-C. Cavard (Cavard, 1994, p. 37) : "Le vieux conflit entre "le rural" et "l'urbain" semble révolu. La dialectique conflictuelle apparaît dépassée et toute une série de complémentarités doivent être recherchées. Une structure élaborée sous forme de PNR pourrait constituer ce "laboratoire" d’idées

8. À titre indicatif, la croissance démographique moyenne en 1990 et 1999 sur le territoire du Parc s'est élevée à +0,91\% (se décomposant en $+0,47 \%$ pour le solde migratoire et $+0,44 \%$ pour le solde naturel). À comparer au taux enregistrés dans la Région Île-de-France : $+0,30 \%$ (dont $-0,06 \%$ résultant du solde migratoire et $+0,24 \%$ du solde naturel).

9. Voir par exemple, la définition qui en est donnée dans le Dictionnaire de Géographie de P. George et F. Verger (référant aux travaux de 1976 de G. Bauer et J.-M. Roux) : « La rurbanisation est l'utilisation par des citadins aux fins d'habitat principal de sites privilégiés, jugés agréables, où la campagne reste visuellement une campagne par l’importance des espaces agricoles, par la faiblesse des densités et des équipements collectifs. Le citadin qui garde son emploi en agglomération urbaine, et donc migre quotidiennement, est plutôt intéressé par les paysages de la campagne que par son organisation sociale». Op. cité p. 411 .

10. Le journal du Parc Couleurs du Vexin signale dans son n 15 (janv-fév.-mars 2002) que sur les 571 exploitations agricoles en 1988, n'en subsistent plus que 399 en 2000 (selon les données du RGA), soit une baisse de 172 (équivalent à -30,1\% sur la période). La base BIEN indique à ce propos que sur les 50 ventes effectuées par des agriculteurs entre 1996 et 2001 , $24 \%$ étaient destinées à de l'habitation et $34 \%$ correspondaient à des terrains constructibles. 
neuves et d'expériences locales innovantes. » Il ajoute plus loin : «À l'heure où l'aménagement du territoire "se fabrique" à une échelle nationale et internationale, les espaces périurbains parisiens seront obligés de "travailler" ensemble. C'est en ce sens que le "local" ou le "territoire" peut retrouver une nouvelle dynamique. En définitive, il n'y a peut-être pas de chose plus difficile à faire que d'aménager l'espace quand on est si près d'une mégalopole. La création du Parc Naturel Régional devrait permettre de relever ce défi ». Les termes de la problématique qui nous préoccupe sont ici clairement exposés. Un PNR se développant aux franges d'une agglomération, peut-il être conçu comme un territoire "sous cloche ", se constituant dans une logique unilatéralement défensive?

\section{Un discours qui privilégie l'environnement et... un passé idéalisé}

Sachant que Couleurs du Vexin est le journal officiel du parc, s'adressant aux résidents de ce territoire dans une logique de communication institutionnelle interne (Rosemberg, 2000), il est pertinent d'en décrypter les thématiques dominantes. Quelle image s'ébauche au fil des articles? Quels aspects sont tout particulièrement mis en avant? Quels autres sont ignorés, voire occultés? Après un premier survol des numéros disponibles, certains thèmes se dégagent avec force. Que la question de l'environnement, entendu dans un sens large (paysages, qualité de l'air, de l'eau, protection de la faune, de la flore) constitue l'un des leitmotivs ne surprendra guère, par contre l'approche environnementale est systématiquement défensive (il s'agit de sauvegarder, éventuellement de restaurer un paysage traditionnel, de défendre une espèce menacée), teintée d'une volonté éducative (transmettre aux jeunes générations le souci environnemental). L'investissement dans les nouvelles énergies n'est quasiment jamais abordé, ni le soutien à des entreprises innovantes dans le domaine écologique. De fait, c’est la patrimonialisation des paysages qui se dégage, la référence à un paysage idéalisé (avec haies naturelles, haies champêtres, arbres isolés, villages " minéraux ", enduits de façade...) se concrétisant lors de l'élaboration des chartes paysagères.

La question de l'histoire locale s'impose également. Dans les premiers numéros, il s'agissait de faire émerger une mémoire locale, d'où l’importance accordée à certains personnages, aux itinéraires emblématiques, et par la suite, ce sont les écomusées ${ }^{11}$ qui prirent une place grandissante. Ceux-ci répondent à la volonté de retrouver des racines, de faire connaître de vieux métiers ou des traditions « authentiquement » vexinoises. De nombreuses fêtes viennent se greffer autour des écomusées, en cela ils attestent de la volonté de faire émerger une «identité vexinoise ». En parallèle à ces manifestations, plusieurs initiatives estampillées « Parc » sont lancées : création d'un "Salon des salons » pour les artistes du Vexin (en 2000), réalisation d’une opération destinée aux enfants intitulée «Imagine ton Parc » (en 2001), la labellisation d'une «Marque Parc » (en 2003).

Si les questions économiques sont abordées, elles mettent d'abord en avant le maintien de la ruralité, le développement des activités touristiques et artisanales. Certes, dans l'avant projet de révision de charte ${ }^{12}$, il est bien stipulé qu' « une ruralité vivante, c'est aussi un territoire économiquement fort. Les Vexinois s'accordent pour reconnaître que le développement économique conditionne le financement des services et des équipements publics qu'ils appellent de leurs vœux; à condition que celui-ci soit compatible avec les objectifs environnementaux du parc, et que réciproquement, un désir exacerbé de protection ne stérilise pas les initiatives d’implantations », mais le décalage entre les intentions affichées et les résultats observés n’est jamais analysé de façon rigoureuse. Le même constat pourrait être fait sur les aspects concernant les dynamiques sociales. L'illusion continue à prévaloir, en partie renforcée par l'affichage d'une ruralité mythifiée dans les pages de Couleurs du Vexin que la rurbanisation ne concerne pas ce territoire... quand tout atteste de l'inverse. Ceci contribue à construire une identité « Parc ».

11. Maison du Pain créée à Commeny en 1998, Musée de la Moisson à Sagy en 2000, musée du Vexin français à Théméricourt en 2001, maison de la Meunerie à Valmondois en 2004, d'autres projets étant par ailleurs programmés : Camp de César à Nucourt, maison des Fruits oubliés à Saint-Clair-sur-Epte et maison de la Nature à Vétheuil.

12. Numéro spécial n² 2, Couleurs du Vexin, novembre 2004. 


\section{La modification du profil social des résidents}

Dans un contexte de faible offre résidentielle nouvelle ${ }^{13}$, la modification du profil social des populations résidentes résulte essentiellement des mobilités résidentielles. Le profil des nouveaux arrivants étant susceptible de modifier, touche par touche, le tissu social initial. Le fait que les ménages soient en outre très majoritairement propriétaires de leur logement (79,3\% en 1999 dans le PNR pour 57,4 \% dans le Val-d'Oise) incite à regarder de manière plus attentive les mobilités des acquéreurs de biens immobiliers, ce que la base BIEN rend envisageable.

La figure 1 fournit une première indication concernant le profil des résidents n'habitant pas dans le même logement lors du recensement précédent et étant donc venus s'installer entre 1990 et 1999. Toutefois, les analyses nécessitent de prendre en compte le poids inégal des différentes CSP parmi les mobiles. Comme l'indique le tableau 1, les employés constituent 31,5\% de ceux-ci dans le Val-d'Oise, quand, a contrario, les cadres et professions intellectuelles supérieures (CPIS) ne pèsent que $13,5 \%$ et les retraités, $8,8 \%$. Les profils des mobiles venus s'installer dans le PNR et le Val-d'Oise peuvent être mis en regard, à la nuance près toutefois que 77 des 94 communes du PNR seulement font partie du Val-d'Oise, les autres appartenant aux Yvelines. Les CPIS et les professions intermédiaires constituent néanmoins des catégories sur-représentées parmi les mobiles du PNR, que ceux-ci aient effectué des mobilités internes ou soient venus de l'extérieur de celui-ci. Il est à noter que les écarts sont moins sensibles entre la ville nouvelle ${ }^{14}$ de CergyPontoise et le PNR, qu'entre ce dernier et le Val-d'Oise pris dans son ensemble.

\begin{tabular}{|c|c|c|c|c|}
\hline $\begin{array}{l}\text { CSP des individus } \\
\text { mobiles }^{*}\end{array}$ & PNR Vexin & Agglo. Cergy.P. & $\begin{array}{l}\text { Val-d'Oise (hors } \\
\text { PNR et Agglo } \\
\text { Cergy-P.) }\end{array}$ & Val-d'Oise \\
\hline $\begin{array}{l}\text { Cadres et professions } \\
\text { intellectuelles } \\
\text { supérieures (CPIS) }\end{array}$ & $17,6 \%$ & $15,6 \%$ & $12,7 \%$ & $13,5 \%$ \\
\hline $\begin{array}{l}\text { Professions } \\
\text { intermédiaires }\end{array}$ & $29,7 \%$ & $27,3 \%$ & $25,4 \%$ & $26,0 \%$ \\
\hline Employés & $24,8 \%$ & $32,6 \%$ & $31,8 \%$ & $31,5 \%$ \\
\hline Ouvriers & $17,7 \%$ & $18,9 \%$ & $20,7 \%$ & $20,2 \%$ \\
\hline Retraités & $10,2 \%$ & $5,6 \%$ & $9,4 \%$ & $8,8 \%$ \\
\hline Total mobiles & 22280 & 53770 & 228563 & 301361 \\
\hline
\end{tabular}

Tableau 1 : CSP des individus mobiles, ne résidant pas dans le même logement, entre 1990 et 1999, dans le PNR du Vexin français, la Ville nouvelle de Cergy-Pontoise et le Val-d'Oise (Source : Fichier IRIS Mig 4. Données INSEE transmises Centre Quételet)

Socio-economic classification of people whose housing has changed between 1999 and 1990, in the Natural Regional Park of le Vexin Français, the New town of Cergy-Pontoise and the Val-d'Oise county

La figure 1 montre par ailleurs que ce sont les portions du PNR les plus proches de la communauté d'agglomération de Cergy-Pontoise qui voient les professions intermédiaires, souvent associées aux CPIS, dominer parmi les mobiles. Les marges rurales plus lointaines laissent par contre apparaître des associations employés-ouvriers plus fréquentes. Ceci révèle une tendance

13. Les données INSEE indiquent qu'il y avait 28722 résidences principales en 1990 sur le territoire de l'actuel PNR et 32992 en 1999. Le poids des résidences principales par rapport à la totalité des logements ayant crû entre $1990(85,5 \%)$ et $1999(88,3 \%)$. Les maisons individuelles représentant en 1999, 87,1\% des résidences principales.

14. Le statut de ville nouvelle a pris fin en décembre 2002 pour Cergy-Pontoise, celle-ci devenant Communauté d'Agglomération à compter du $1^{\text {er }}$ janvier 2004. 


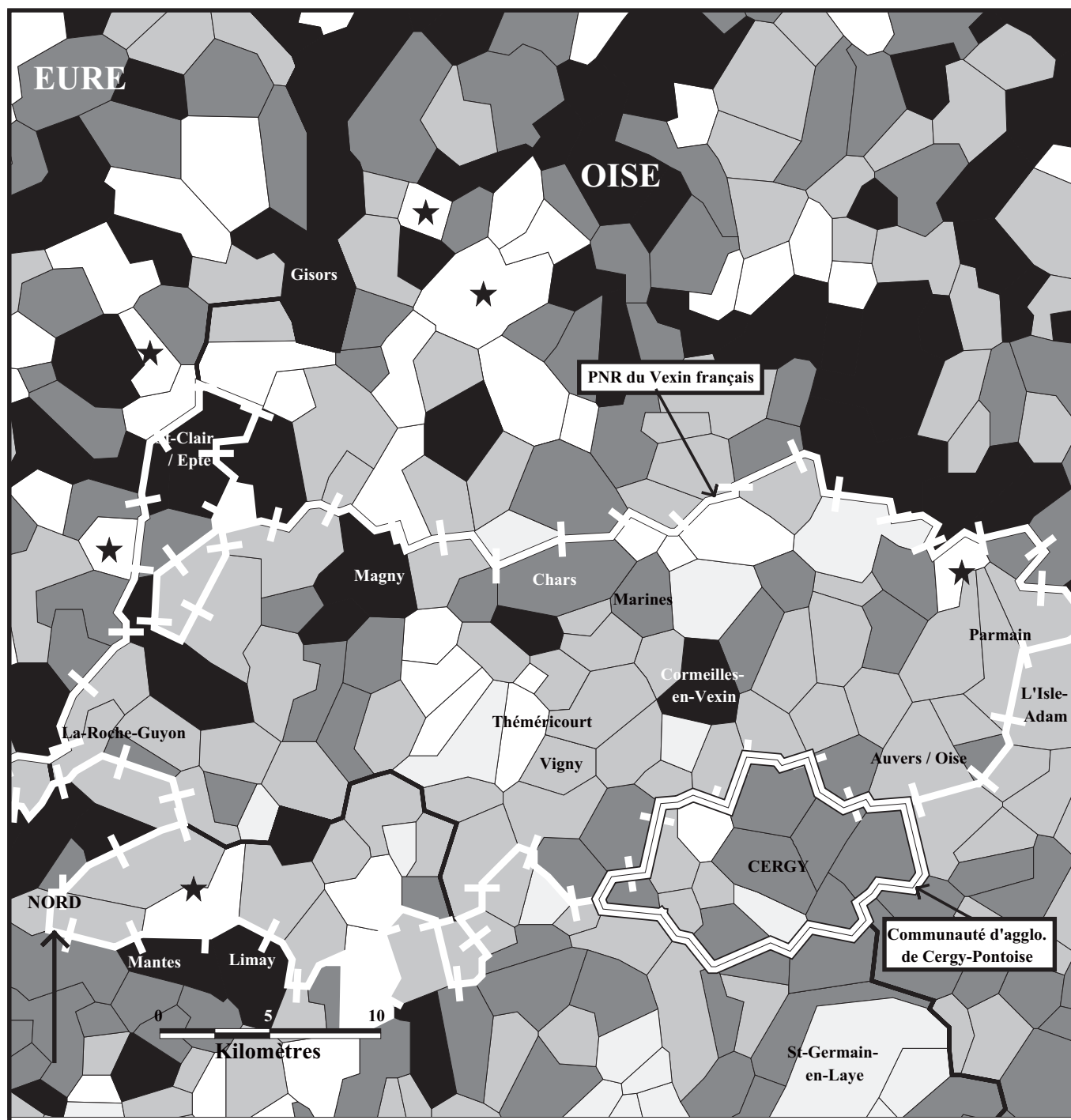

CSP LA PLUS REPRESENTEE PARMI LES NOUVEAUX ARRIVANTS ENTRE 1990 ET 1999. CHOIX POSSIBLE ENTRE "CPIS", "PROF. INTERM.", "EMPLOYÉS", "OUVRIERS", "RETRAITÉS".

Source : Fichier IRIS MIG4. Extraction au 1/4. Données INSEE transmises par le Centre Quetelet.

- CSP arrivant en tête parmi les résidents de 1999,

ne résidant pas dans le "même logement" en 1990 :
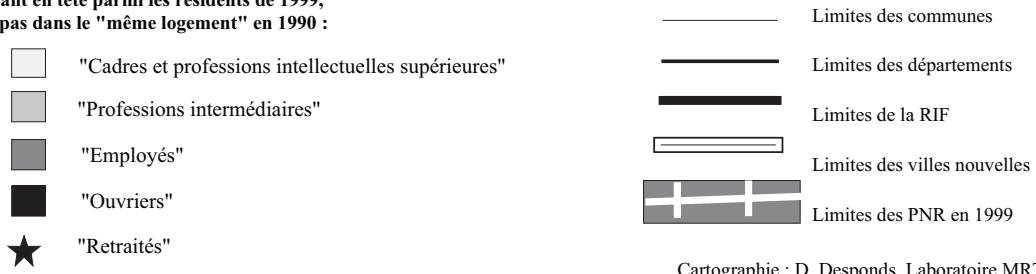

Cartographie : D. Desponds. Laboratoire MRTE UCP.

Figure 1 : CSP la plus représentée parmi les nouveaux arrivants entre 1990 et 1999

Most frequent social activity among the new residents settled between 1990 and 1999 
à la « spécialisation sociale » des territoires en fonction d'un gradient centre-périphérie (Berger, 2004 ; Desponds, 2005). Toutefois ces phénomènes ne peuvent être réduits à un effet distance au centre, les écarts entre certains secteurs des territoires urbains tendant également à se renforcer. A titre indicatif, l'est du Val-d'Oise enregistre une sur-représentation de l'association employésouvriers parmi les mobiles, l'offre résidentielle fortement axée sur l'habitat collectif à tonalité sociale jouant là un rôle crucial.

La base BIEN ${ }^{15}$ offre un éclairage complémentaire sur la question des mobilités résidentielles, en particulier sur les aspects concernant les provenances géographiques et le profil social des acheteurs de biens immobiliers dans le PNR (pour la seule partie Val-d'Oise, les données des Yvelines ne nous ayant pas été communiquées). La figure 2 permet ainsi de mettre en évidence l'aspect sectoriel des mobilités effectuées. Les mobilités internes au PNR représentent certes $31,55 \%$ des transactions effectuées, mais ce sont surtout des ménages résidant initialement sur un axe Paris - Cergy-Pontoise qui forment l'essentiel des acquéreurs, dans une logique d'éloignement du centre.

La comparaison entre le profil social des acheteurs dans le PNR et dans le Val-d'Oise (fig. 3), compte tenu du profil social des vendeurs, permet de mettre en lumière les logiques de la « substitution sociale »(Desponds, 2005) sur ces territoires. Si des processus de reproduction socio-résidentielle sont observés ${ }^{16}$, de sensibles écarts apparaissent également selon les entités de référence. Quelle que soit la CSP du vendeur, les CPIS et dans une moindre mesure les professions intermédiaires sont ainsi sur-représentés dans le PNR Le phénomène inverse pouvant être observé pour les ouvriers et dans une moindre mesure les employés. Ceci contribue à modifier le tissu social initial de ces territoires. L'incidence sur les prix des transactions renforce en outre les mécanismes de « filtrage social », certains territoires devenant, compte tenu de l'appréciation immobilière, plus difficilement accessibles aux catégories disposant du plus faible « capital spatial ${ }^{17}$ ».

Les indicateurs fiscaux ${ }^{18}$ offrent un éclairage complémentaire (Sagot, 2001) sur la répartition des ménages selon leur profil social. De nombreuses études confirment par ce biais une tendance au renforcement des inégalités territoriales. Ces processus peuvent en partie s'interpréter comme la résultante des dynamiques résidentielles. Ainsi les communes où les «substitutions sociales » conduisent à un renforcement des catégories aisées devraient voir leur profil fiscal s'améliorer et le taux de non imposés décroître et inversement dans le cas des communes caractérisées par l'arrivée de populations à faibles ressources.

La comparaison des revenus bruts moyens de 1994 et de $1999^{19}$, tenant compte de l'inflation cumulée sur la période, indique de sensibles disparités entre les ménages fiscaux du PNR et ceux du Val-d'Oise. Augmentation de $+2,44 \%$ dans le premier cas, baisse de $-0,85 \%$ dans le second. La ville nouvelle de Cergy-Pontoise, à proximité même du PNR, enregistre une baisse significative de $-1,91 \%$, en partie imputable à la sur-représentation sur son territoire de jeunes ménages, de

15. Alimentée par les transactions immobilières déclarées par les notaires, elle constitue une base particulièrement riche permettant de connaître le profil (social, matrimonial, nationalité, âge, mais, on peut le regretter l'absence d'indications sur la structure familiale) des vendeurs comme des acheteurs. Dans ce dernier cas, des informations sur le lieu de résidence antérieur peuvent aisément être exploitées. En outre, y figurent de nombreuses indications sur les spécificités des biens acquis. À titre indicatif, cette base, pour le seul Val-d'Oise, comportait 60899 transactions (mais seules 41095 transactions

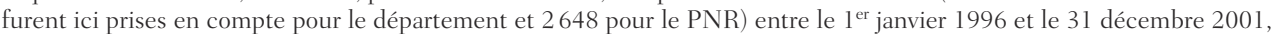
couvrant donc la période de mise en place du PNR du Vexin français.

16. Si l'on excepte les retraités, c'est le plus souvent quand une catégorie sociale vend que sont observés les taux les plus élevés d'acheteurs de cette même catégorie. Ce qui ne signifie pas que celle-ci soit classée comme la première catégorie d'acheteurs. Se retrouvent ici les effets de l'inégale distribution des CSP. Comme l'indique la figure 2, dans le Val-d'Oise, les professions intermédiaires constituent $29,98 \%$ des acheteurs, les ouvriers seulement $13,63 \%$.

17. Entendu comme la compétence à se mouvoir dans l'espace et à accéder aux lieux les plus fortement valorisés, ceux qui possèdent le plus fort « capital territorial ». Le «capital spatial » résulte à la fois de moyens financiers et de capacités à appréhender les mécanismes du marché immobilier (Desponds, 2004).

18. N’ont été retenus ici que le taux de ménages fiscaux non imposés sur la commune et les revenus bruts moyens déclarés par ces mêmes ménages, c'est-à-dire les revenus avant impôts.

19. Autrement dit des revenus gagnés lors des années civiles 1993 et 1998, période correspondant au début de l'existence du PNR du Vexin français. 


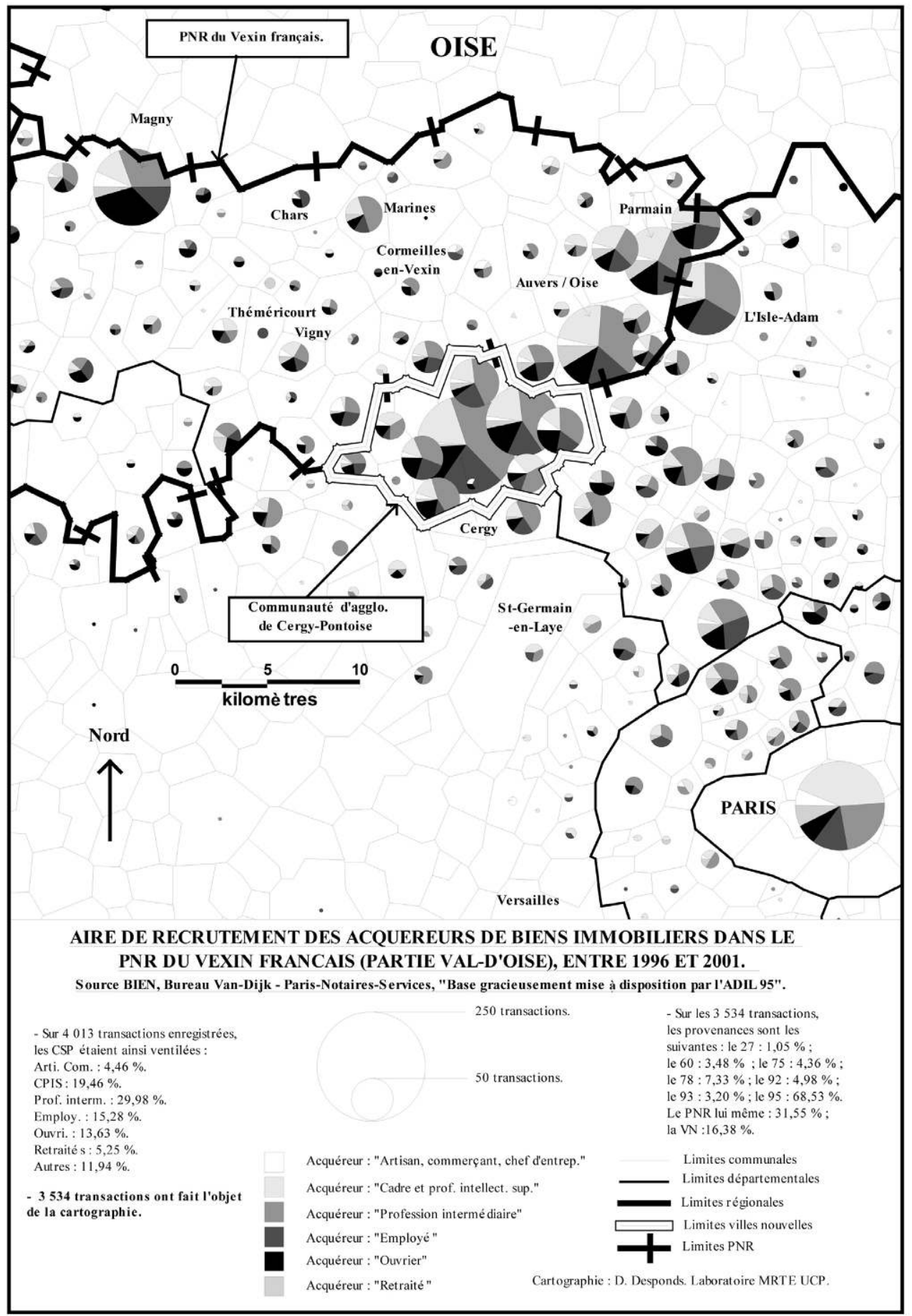

Figure 2 : Aire de recrutement des acquéreurs de biens immobiliers dans le PNR du Vexin français (partie Val-d'Oise), entre 1996 et 2001

Catchment area of estate buyers in the Natural Regional Park of le Vexin français (Vald'Oise part only), between 1996 and 2001 


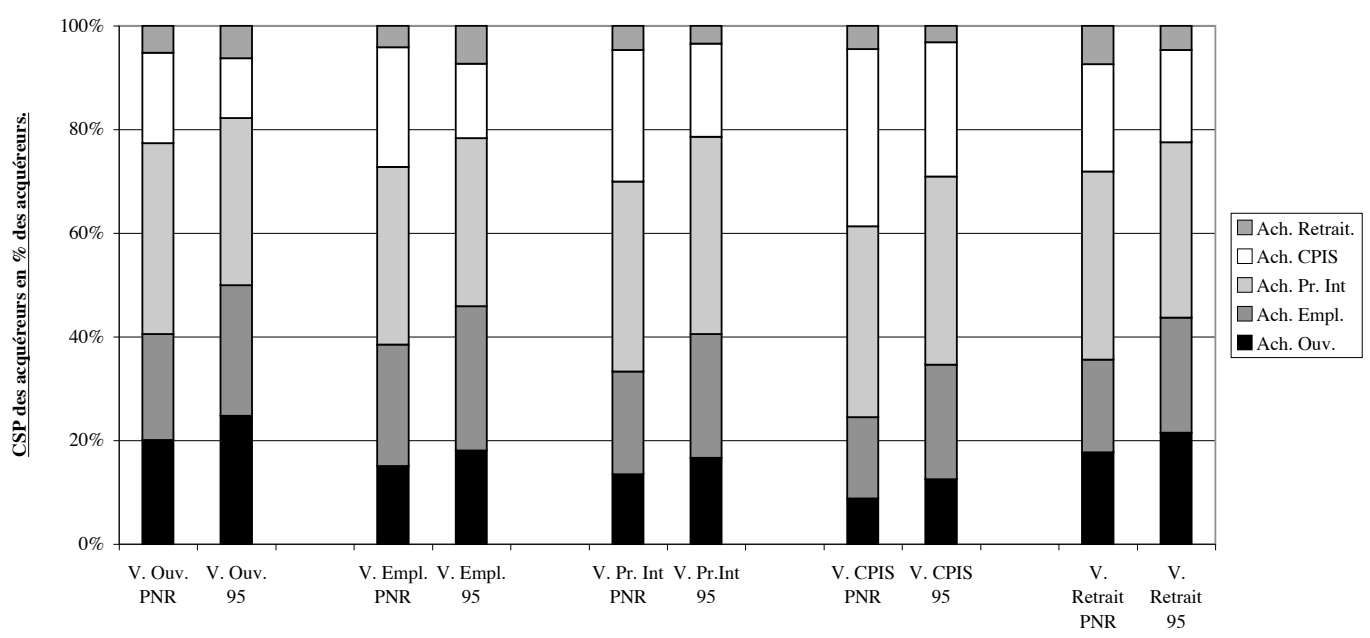

C.S.P. des vendeurs de biens immobiliers dans chacun des territoires.

Figure 3 : Profils comparés des CSP des acquéreurs de biens immobiliers, entre 1996 et 2001, en fonction du profil des vendeurs, dans le Val-d'Oise et le PNR du Vexin français

Comparison between the social profile of estate buyers and the social profile of estate sellers in the Natural Regional Park of le Vexin français and Val-d'Oise county (1996 and 2001)

ménages plus modestes (Korsu, 2001) et de familles monoparentales. La forte appréciation observée dans le PNR du Vexin français doit être nuancée, des résultats beaucoup plus nets ayant été enregistrés dans le PNR de la Haute Vallée de Chevreuse $(+10,15 \%$ sur la même période quand la Ville Nouvelle de Saint-Quentin ne connaissait une augmentation que de $+1,26 \%$ ). La tendance ici dégagée est confirmée par le poids respectif des non imposés sur chacun des territoires et les évolutions enregistrées sur la même période d'étude. La tendance de fond laisse apparaître une baisse générale de ces taux, mais avec des écarts d'amplitude : de 34,76\% à 31,16\% pour le PNR du Vexin français, là aussi à comparer aux niveaux atteints dans le PNR de la Haute vallée de Chevreuse (de 25,55 \% à 22,47 \%). Le Val-d'Oise n'enregistre qu'une baisse légère (de 37,56 \% à $37,15 \%$ ), assez similaire à celle de la ville nouvelle de Cergy-Pontoise (de 38,85\% à 38,52\%).

Ces indications ne fournissent que des tendances. La période d'étude est trop brève pour conclure avec certitude sur le rôle que le PNR joue quant à la «spécialisation sociale » des territoires. Toutefois, ces disparités fiscales venant s'ajouter aux spécificités des mobilités résidentielles différenciées observées par ailleurs, offrent un argument supplémentaire et convergent : un processus d'embourgeoisement existe sur ce territoire. Il s'est probablement amorcé avant même la mise en place du Parc. Le profil social des populations résidentes tend à se distinguer au sein du département et par rapport à la communauté d’agglomération voisine de Cergy-Pontoise. Ceci ne constituait pas le but initialement recherché, il s'agit d'une conséquence des mécanismes restrictifs concernant l'offre résidentielle ${ }^{20}$, de l'augmentation sensible des prix de l'immobilier ${ }^{21}$ en particulier dans le cas des

20. Signalons à ce propos que la question de l'habitat social n'est jamais abordée dans le journal «Couleurs du Vexin ", à l'exception notable du n 3 de décembre 1997, où un article intitulé : «Locatif social, mythe ou réalité? » a été rédigé par F. Marchant, la directrice du PACT-ARIM 95.

21. La base BIEN nous permet de dégager des indications sur les prix moyens des transactions effectuées. Ainsi, pour les 1689 maisons acquises entre 1996 et 2001 dans le PNR le prix en euros constants passe de 1173 euros $/ \mathrm{m}^{2}$ en 1996 à 1381 euros $/ \mathrm{m}^{2}$ en 2001 (soit une croissance de $+17,73 \%$ ). L'appréciation est beaucoup plus marquée pour les 211 appartements acquis sur ce territoire (1315 euros $/ \mathrm{m}^{2}$ en 1996 à 1791 euros $/ \mathrm{m}^{2}$ en 2001 , soit une croissance de $\left.+36,2 \%\right)$. À titre indicatif, dans le même temps, les acquisitions de maisons dans le Val-d'Oise enregistraient les évolutions suivantes (de 1341 euros $/ \mathrm{m}^{2}$ à 1540 euros $/ \mathrm{m}^{2}$, soit une croissance de $15,6 \%$ ) et les appartements (de 1301 euros $/ \mathrm{m}^{2}$ à 1291 euros $/ \mathrm{m}^{2}$ soit une baisse de -0,9\%). Il faudrait cependant affiner les analyses selon les tailles des biens et leur date de construction, ces éléments ayant une forte incidence sur les niveaux de prix enregistrés. 
appartements et de l'image de ce territoire. Perçue comme positive, elle contribue à influencer les stratégies résidentielles déclenchées par les ménages, cette tendance étant d'autant plus marquée que les ménages ont des enfants. Les effets induits par sa mise en place incitent à appréhender un PNR comme un mode de « sélection douce » des populations résidentes.

\section{Une dépendance économique accrue vis-à-vis des pôles périphériques}

La question de l'emploi figure explicitement dans la charte du PNR ${ }^{22}$, toutefois, comme le stipule l'article 27 : «Il est dans la mission du Parc d'être particulièrement attentif à tout projet économique qui serait contraire aux engagements affichés en matière de protection et de sauvegarde de l'environnement et de rechercher une solution compatible avec les deux objectifs de protection de l'environnement et de développement économique ». Il s'agit donc de permettre un développement économique compatible avec les engagements pris sur le plan environnemental. Les activités faisant l'objet d'une attention soutenue sont les activités touristiques et celle de type artisanal. Le nombre d'emplois ${ }^{23}$ dans le périmètre du Parc a décrû entre 1990 et 1999, passant de 19236 à 17443, quand dans le même temps les actifs résidant sur ce territoire sont devenus plus nombreux, passant de 32844 à 34891 . Ceci génère automatiquement une détérioration du ratio « actifs au lieu de travail/actifs au lieu de résidence » (de 0,59 à 0,51). Le PNR a donc vu se renforcer son profil de « territoire dortoir », les migrations pendulaires s'en retrouvant intensifiées. Phénomène par ailleurs accentué par la réduction des actifs stables ${ }^{24}$, ceci ne constitue en aucun cas une spécificité de ce territoire (Ascher, 1995; Allard et al., 1997).

De sensibles écarts sont observés selon les CSP comme le montre la figure 4. Les CPIS constituent d'une façon générale la catégorie connaissant les plus fortes dissociations lieu de domicile - lieu de travail. La figure 5 montre par ailleurs que la dépendance économique à l'égard de Cergy-Pontoise tend à s'accentuer ${ }^{25}$. En 1990, 6079 actifs résidant sur le territoire du Parc y travaillaient, soit $18,51 \%$, ils sont devenus 7452 en 1999, soit 21,36\%. Dans le même temps les actifs travaillant dans le Parc ont vu leur nombre fortement chuter, passant de 11640 (soit 36,05\%) à 8909 (soit $25,53 \%$ ) à peine plus que Cergy-Pontoise. Tenant compte des dynamiques économiques en cours dans le Parc, de l'écart entre les emplois disponibles et le profil social des actifs résidents ${ }^{26}$, dans un contexte favorable à l'accentuation des mobilités liées à l'emploi (Lévy, 2000; Kaufmann et Jemelin, 2004), il est fort probable que les tendances ne se renforcent : le PNR devenant une «périphérie protégée » du pôle périphérique que constitue Cergy-Pontoise au sein du système francilien.

Il est en outre à signaler que les pôles d'emploi éloignés des axes structurants Paris - CergyPontoise ou Paris - vallée de la Seine, ne jouent qu'un faible rôle, ainsi de Roissy qui concentre pourtant 22,38 \% des emplois du Val-d'Oise. Les pôles d'emploi extérieurs à la région Ille-de-France (Beauvais, Gisors) n'exercent guère d'attraction déterminante. Au final, des similitudes fortes apparaissent entre les aires de recrutement des acquéreurs de biens immobiliers dans le PNR et les aires d'attraction des pôles d'emploi, en résultante des logiques de prospection résidentielle mise en œuvre par les ménages, compte tenu des contraintes liées à l'emploi.

22. Article 22 : «Développer l'activité agricole»; article 23: «Améliorer les conditions de l'activité agricole »; article 25 : «Animer le développement économique »; article 26: «Valoriser les potentialités locales »; article 27 : « Implanter les activités économiques »; article 28 : « Mettre en place des zones d’activités intercommunales avec péréquation de taxes »; article 29 : «Maintenir et dynamiser les commerces et l'artisanat. »

23. D'après les indications des fichiers MIRABELLE 1990 et MOBILITE 1999 fournis par l'INSEE. Il faut cependant préciser qu'à des fins de comparaisons ont été exclus les « micro-flux », ceux inférieurs strictement à 3 actifs de commune à commune, ce qui, dans le cas d'un territoire rural, génère une sensible sous évaluation, de l'ordre de $15 \%$ pour les actifs au lieu de résidence, de $20 \%$ pour ceux au lieu de travail.

24. En 1990, 35,44 \% des actifs travaillaient et résidaient sur ce périmètre, mais seulement 25,53 \% en 1999.

25. Aspect qui n'est d'ailleurs jamais évoqué dans le journal Couleurs du Vexin qui le plus souvent maintient l'illusion d'un PNR « autonome » sur le plan des activités économiques.

26. En reprenant les informations de la figure 5, les ouvriers représentent 19,71\% des actifs au lieu de résidence, mais 32,37\% des actifs au lieu de travail du PNR, a contrario, pour les CPIS : 17,37\% des actifs au lieu de résidence, mais seulement $10,21 \%$ de ceux au lieu de travail. 


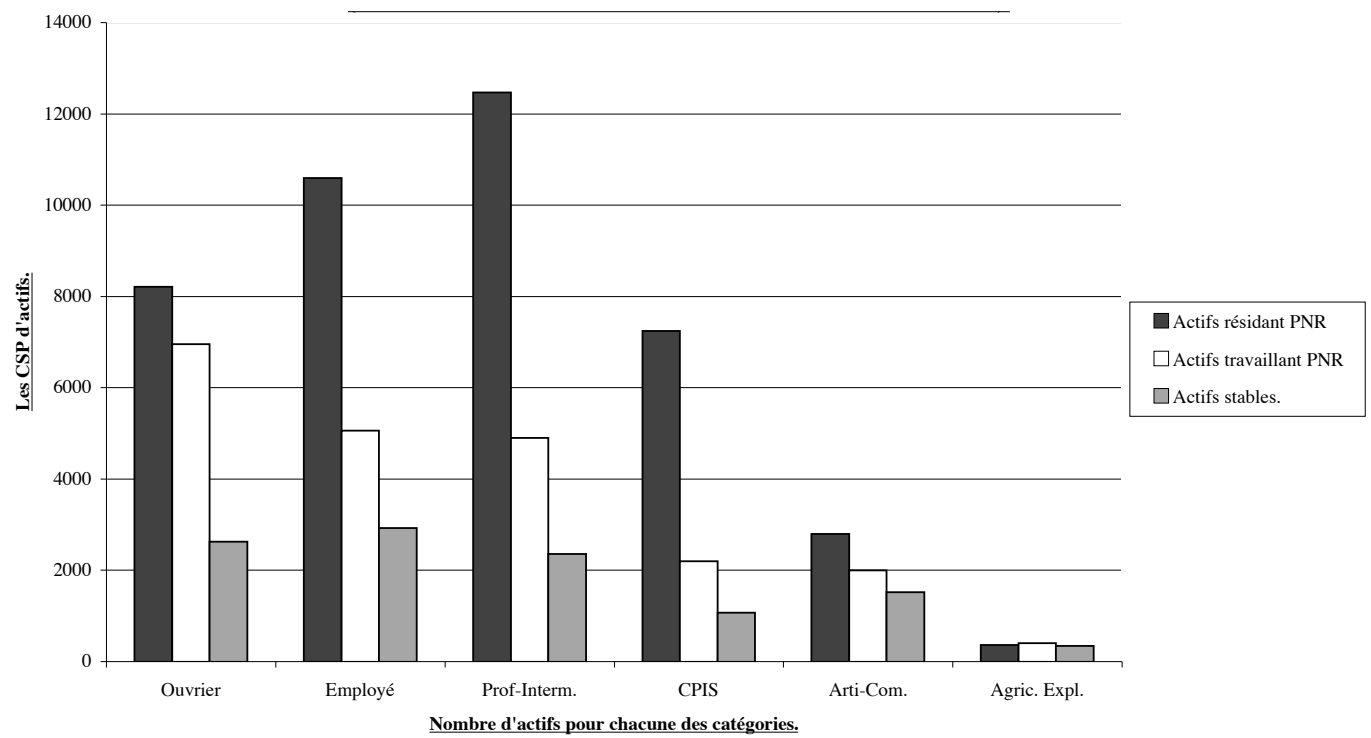

Figure 4 : Actifs travaillant ou (et) résidant dans le PNR du Vexin français, en fonction de la CSP, en 1999 Working age population working and (or) living in the Natural Regional Park of le Vexin français, according to socio economic classifications (1999)

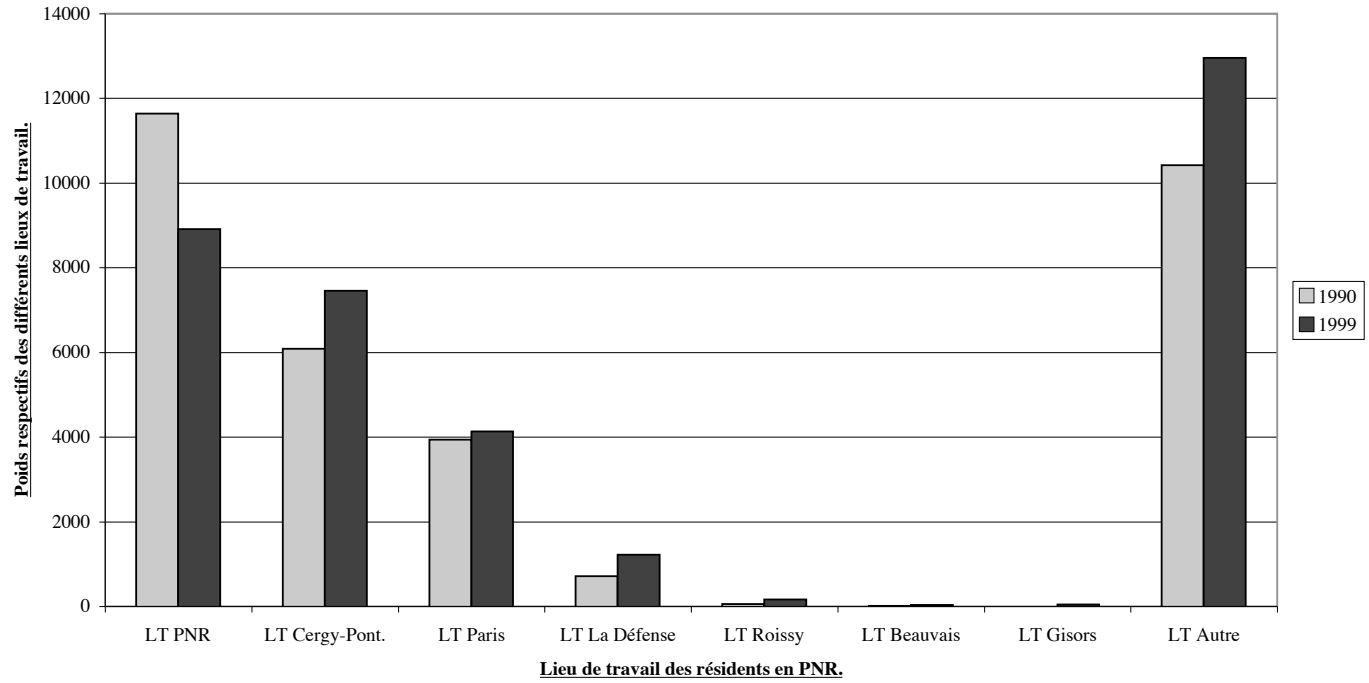

Figure 5 : Lieu de travail des actifs résidant dans le PNR du Vexin français en 1990 et 1999 Places of work of working age population living in the Natural Regional Park of le Vexin français (1990 and 1999) 


\section{Conclusion}

Au-delà d'une rhétorique convenue sur l'identité vexinoise, se traduisant par une volonté de préservation des paysages ou du maintien d'activités traditionnelles, le vaste PNR du Vexin français est confronté, de façon accélérée depuis une dizaine d'années, à de profondes mutations sociodémographiques (fig. 6).

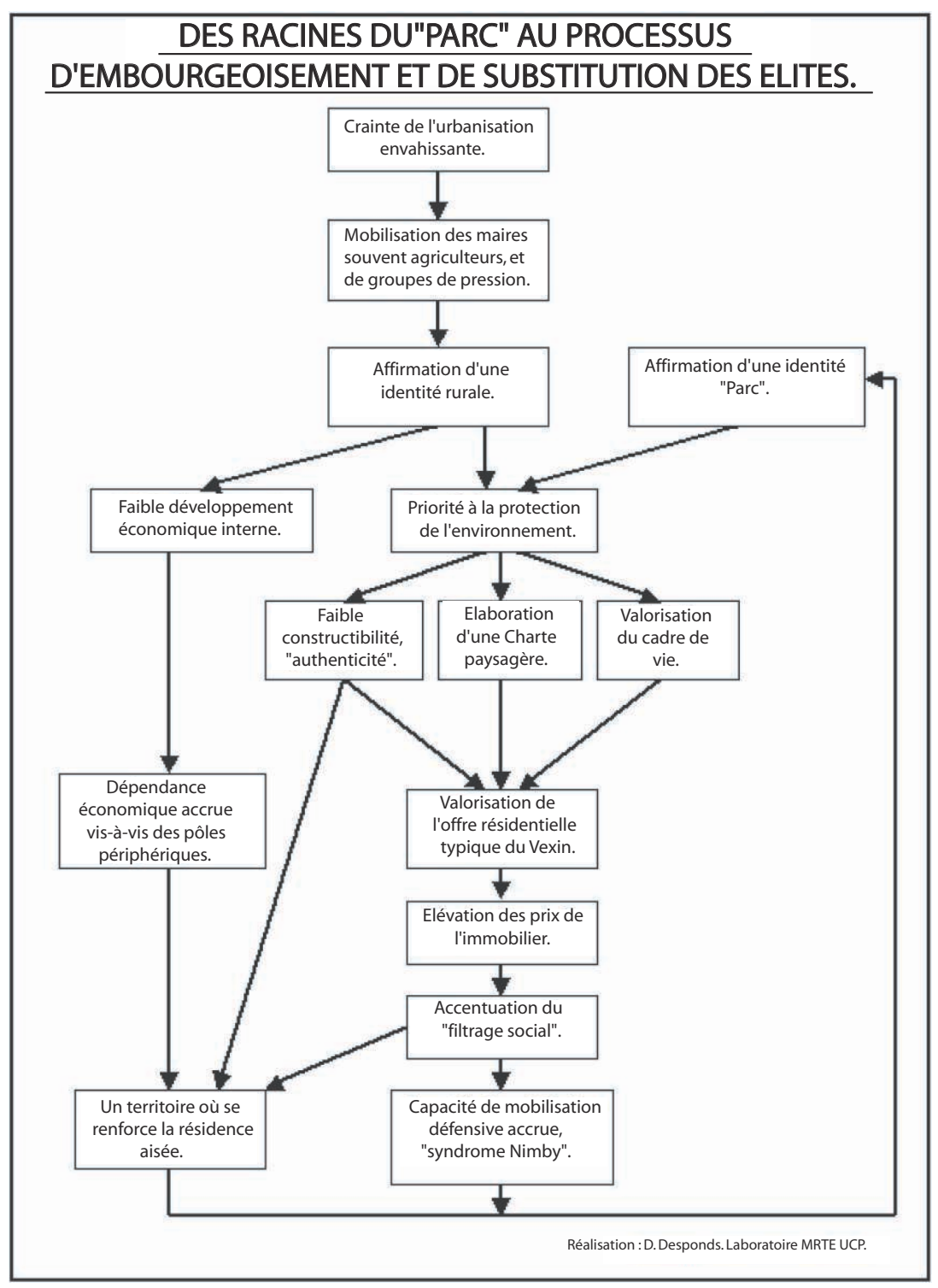

Figure 6: Des racines du «Parc» au processus d'embourgeoisement et de substitution des élites a substitution

From the birth of the "Park" to the gentrification process and to the new elites: 
Son cadre " préservé » aux marges mêmes du tissu urbain de l'agglomération parisienne semble séduire de plus en plus fortement des ménages au profil aisé dont l'ancrage professionnel se situe à l'extérieur du PNR. De façon polémique, on est en droit de s'interroger sur les effets indirects des mesures strictes mises en place dans le domaine des réglementations environnementales. Ne s'agit-il pas aussi, en restreignant de façon drastique l'offre résidentielle, de se doter d'outils efficaces, mais politiquement acceptables, contribuant au renforcement de l'entre-soi et de la mise à distance des populations « indésirables »? Les orientations qui seront définitivement adoptées par la nouvelle Charte, courant 2007, fourniront rapidement des indications quant aux perspectives sociales de ce territoire. A contrario, il est indubitable que l'absence d'un tel PNR accélèrerait les processus de périurbanisation sous forme de lotissements. Les restrictions urbanistiques induites par le PNR tendent ainsi à renforcer la densification urbaine des pôles périphériques, en limitant «l'hémorragie résidentielle » à laquelle ils pourraient être confrontés. Ceci incite à invalider toute approche géographique qui ne prendrait pas en compte les interactions complexes entre les territoires, dans une logique systémique. Compte tenu de ces différents aspects, les PNR périurbains contribuent à faire émerger une figure d'un type nouveau dans le contexte des dynamiques plus générales concourant à la périurbanisation, celle des marges protégées en cours d'embourgeoisement.

\section{Bibliographie}

Allard (M.), Beaucire (F.), Emangard (P.-H.), 1997. - « Les déplacements domicile-travail dans l'archipel nantais », Transports Urbains, n 96, juil.-sept. 1997, p. 5-13.

Ascher (F.), 1995. - Métapolis, ou l'avenir des villes, O. Jacob, Paris, 345 p.

Bauer (G.), Roux (J.-M.), 1976. - La rurbanisation ou la ville éparpillée, Paris, Seuil, 189 p.

Berger (M.), 2004. - Les périurbains de Paris, Paris, CNRS, 317 p.

Cavard (J.-C.), 1994. - « Histoire de la création du Parc naturel régional du Vexin français », Publication des Amis du Vexin Français, n 34, mai 1994, p. 23-38.

DESPONDS (D.), 2003. - Stratégies résidentielles, différenciation spatiale et recomposition sociale : application à l'aire d'influence de la «Ville Nouvelle » de Cergy-Pontoise, thèse de doctorat, Université de Cergy-Pontoise, décembre 2003, 860 p.

Desponds (D.), 2004. - «Contribution des démarches prospectives différenciées au renforcement des logiques ségrégatives; exploration dans l'aire d'influence de la "Ville Nouvelle" de Cergy-Pontoise », Communication au Colloque Espaces et sociétés aujourd'hui, la géographie sociale dans les sciences et dans l'action, Rennes, 21-22 octobre 2004, 21 p.

Desponds (D.), 2005. - «Stratégies résidentielles et logiques ségrégatives. Investigations dans l'aire d'influence de Cergy-Pontoise ", Paris, Connaissances et savoirs, 760 p.

Dodier (R.), 2006. - « Solidarités sociales et territoriales », Travaux et Documents n² 24, ESO, p. 15-22.

DonZelot (J.), 2004. - « La ville à trois vitesses : gentrification, relégation, périurbanisation », Esprit n 303 , p. 14-39.

Estebe (P.), 2004 - « Quel avenir pour les périphéries urbaines », Esprit, n 303, p. 82-96.

George (P.), Verger (F.), 2000. - Dictionnaire de la Géographie, Paris, PUF, 500 p.

JAILLET (M.-C.), 2004. - «L'espace périurbain, un univers pour les classes moyennes », Esprit, n³ 303, p. 40-62.

Kaufmann (V.), Jemelin (C.), 2004 - « La motilité, une forme de capital permettant d'éviter les irréversibilités socio-spatiales », Communication au Colloque Espaces et sociétés aujourd'hui, la géographie sociale dans les sciences et dans l'action, Rennes, 21-22 octobre 2004, 10 p.

Korsu (I.), 2001. - Les dynamiques d'inégalités et de pawreté dans les villes nowvelles d'Tlle-de-France, EPA Marne, IAURIF, $178 \mathrm{p}$. 
LARCheR (G.), 1998. - Les terroirs urbains et paysagers, Paris, La Documentation française, coll. « Les rapports du Sénat », $97 \mathrm{p}$.

Levy (J.), 2000. - « Les nouveaux espaces de la mobilité », dans Bonnet (M.), DesjeuX (D.) (dir.), Les territoires de la mobilité, Paris, PUF, p. 150-170.

PnR du VeXin Français, 1995. - Charte du Parc, 9 mai 1995, 104 p.

Rosemberg (M.), 2000. - Le marketing territorial en question, Paris, Anthropos/Economica, coll. «Villes », $184 \mathrm{p}$.

SAGOT (M.), 2001. - «Les évolutions récentes des inégalités en Île-de-France », Les Cahiers de l'habitat, n²9, Paris, IAURIF, p. 7-12.

Cet article a été reçu le 30 juin 2006 et définitivement accepté le 15 janvier 2007. 